\title{
Longitudinal spin transport in diluted magnetic semiconductor superlattices: The effect of the giant Zeeman splitting
}

\author{
Kai Chang* and J. B. Xia \\ National Laboratory for Superlattices and Microstructures, Institute of Semiconductors, Chinese Academy of Sciences, \\ P.O. Box 912, 100083, Beijing, China \\ F. M. Peeters ${ }^{\dagger}$ \\ Departement Natuurkunde, Universiteit Antwerpen (UIA), Universiteitsplein 1, B-2610 Antwerpen, Belgium
}

(Received 21 February 2001; revised manuscript received 26 November 2001; published 11 April 2002)

\begin{abstract}
Longitudinal spin transport in diluted magnetic semiconductor superlattices is investigated theoretically. The longitudinal magnetoconductivity (MC) in such systems exhibits an oscillating behavior as function of an external magnetic field. In the weak magnetic-field region the giant Zeeman splitting plays a dominant role that leads to a large negative magnetoconductivity. In the strong magnetic-field region the MC exhibits deep dips with increasing magnetic field. The oscillating behavior is attributed to the interplay between the discrete Landau levels and the Fermi surface. The decrease of the MC at low magnetic field is caused by the $s-d$ exchange interaction between the electron in the conduction band and the magnetic ions. The spin polarization increases rapidly with increasing magnetic field and the longitudinal current becomes spin polarized in strong magnetic field. The effect of spin-disorder scattering on MC is estimated numerically for low magnetic fields and found to be neglectible for our system.
\end{abstract}

DOI: 10.1103/PhysRevB.65.155211

PACS number(s): 75.50.Pp, 73.50.Jt, 75.30.Et

\section{INTRODUCTION}

The most striking phenomena in semiconductor quantum structures is the tremendous change of the optical and transport properties induced by the quantum confinement effect. The use of diluted magnetic semiconductors (DMS) in such systems provides us with an additional degree of freedom to engineer the optical and transport properties by applying an external magnetic field. ${ }^{1}$ An external magnetic field magnetizes the magnetic ions, which gives rise to the exchange field acting on the electron spin. This spin-dependent energy shift is comparable to the band offset in a DMS superlattice, therefore, influencing significantly the optical property of DMS. The optical properties of the DMS systems have been studied extensively in the past few years. Time-resolved photoluminescence (PL) of a dilute magnetic semiconductor superlattice has shown the feasibility of the spin-alignment mechanism. ${ }^{2-4}$ The strong $s-d$ exchange interaction between the electron spin in the conduction band and the localized magnetic ions gives rise to unique magneto-optical properties. ${ }^{5-7}$ Giant Zeeman splitting, excitonic magnetic polaron, Faraday rotation, and optically induced magnetization are well-known examples. Recent experiments demonstrated that spin-polarized transport in diluted magnetic semiconductors and spin coherence can be maintained over large distances $(\geqslant 100 \mu \mathrm{m})$ and for long times $\left(10^{-9}-10^{-8} \mathrm{~s}\right)$ in metals and semiconductors and showed that the spin of the electron offers unique possibilities for quantum computation and information transmission. ${ }^{8-11}$

One of the fascinating effects of magnetic fields on the electron-transport properties in bulk materials is the wellknown Shubnikov-de Haas (SdH) effect, i.e., magnetoconductivity (MC) or magnetoresistance of the system is independent of the magnetic-field strength at very low magnetic field, and exhibits an oscillating dependence with the magnetic-field strength at higher magnetic fields. ${ }^{12}$ This phenomena arises from the interplay between the quantized Landau levels and the Fermi energy. In semiconductor superlattices the $\mathrm{SdH}$ effect displays a rich diversity of prominent phenomena since the electron motion along the magnetic field is quite different from that in bulk materials. The conductivity of a system is determined by the number of different states near the Fermi energy, the group velocity associated with them, and the coupling of these states to each other by scattering mechanisms. Polyanovskii ${ }^{13}$ presented a theory to describe longitudinal magnetotransport in semiconductor superlattices using the semiclassical approach. A single-band tight-binding model was used to describe the superlattice at very high and very low temperature. Datars and Sipe ${ }^{14}$ extended the theory to the multiple miniband case, and found multiple miniband oscillations in the regime where the second miniband is populated.

In this paper we focus on the effect of the giant Zeeman splitting on the longitudinal magnetoconductivity in DMS superlattices and take as an example the $\mathrm{ZnSe} / \mathrm{Zn}_{0.96} \mathrm{Mn}_{0.04} \mathrm{Se}$ superlattices (see Fig. 1). In a DMS system, the giant Zeeman splitting induced by the $s-d$ exchange interaction is comparable to the band offset, and, therefore, can change significantly the energy spectrum of the minibands and the group velocity, which influences the magnetoconductivity. Here we extend the treatment of the $\mathrm{SdH}$ effect from ordinary semiconductor superlattices to diluted magnetic semiconductor superlattices. We find a spin-dependent conductivity when an external magnetic field is applied along the growth direction. The magnetoconductivity decreases significantly with increasing magnetic field at low magnetic fields, and exhibits an oscillating behavior in strong magnetic fields. A strong spin-polarized current is found with increasing magnetic field. The underlying physics arises from the $s-d$ exchange interaction between the intinerant electron and the 

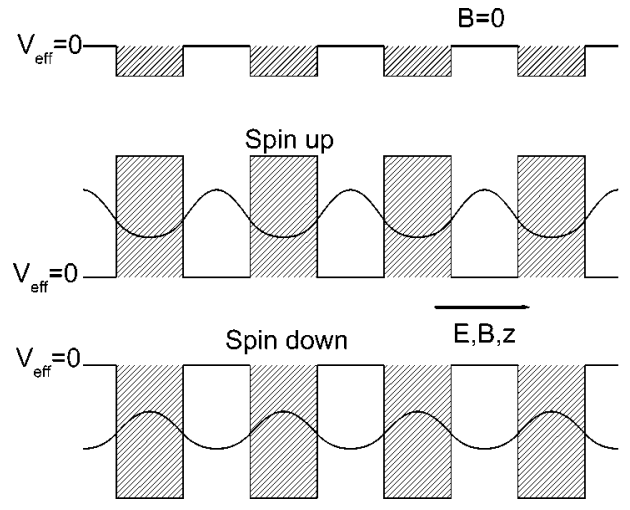

FIG. 1. Schematic illustration of a $\mathrm{ZnSe} / \mathrm{Zn}_{0.96} \mathrm{Mn}_{0.04} \mathrm{Se} \mathrm{DMS}$ superlattice subjected to a perpendicular magnetic field. The shaded regions denote the diluted magnetic semiconductor layers. (a) shows the potential profile for an electron in a DMS superlattice in the absence of a magnetic field. (b) and (c) show the potential profiles for the spin-up and spin-down electron in the presence of a magnetic field, respectively. The probabilities for the spin-up and spin-down electrons are also shown in the figure.

localized magnetic impurity that lifts the degeneracy of the spin-up and spin-down electron band states. Here we neglect the effect of the spin-flip process ${ }^{15}$ and focus only on the effect of the giant Zeeman splitting caused by the $s$ - $d$ exchange interaction on the MC in a DMS superlattice for electrical transport along the growth axis. The molecular-field approximation is used in the present paper that has been shown to lead to an excellent agreement with $\mathrm{SdH}$ measurements performed on $\mathrm{Cd}_{1-x} \mathrm{Mn}_{x} \mathrm{Te} / \mathrm{Cd}_{y} \mathrm{Mg}_{1-y} \mathrm{Te}$ quantum wells (Fig. 4 in Ref. 16). Our numerical results also show that the spin-disorder scattering process is not the dominant scattering mechanism in this case.

The paper is organized as follows, the theoretical model is described in Sec. II, and the numerical results and discussions are given in Sec. III. Finally, the conclusion is presented in Sec. IV.

\section{THEORETICAL MODEL}

We model a DMS superlattice as a periodic array of square potential wells and nonmagnetic barriers and assume that the magnetic ions are distributed homogeneously in the DMS layers (see Fig. 1). In a DMS superlattice, a small external magnetic field gives rise to a giant Zeeman splitting of the conduction-band states, and results in striking differences between spin-up and spin-down electron states of the system. This giant Zeeman splitting arises from the spins of the injected electrons interacting with the $S=5 / 2$ spins of the localized $3 d^{5}$ electrons of the $\mathrm{Mn}^{2+}$ ions via the $s-d$ exchange interaction. ${ }^{5}$ Our theory is based on the assumption that the electron motion in the DMS superlattice can be well described by the effective-mass approximation that is confirmed by recent experiments. ${ }^{16,17,18}$ As shown in Fig. 1, the model Hamiltonian for electrons in such a system is

$$
\begin{aligned}
H= & p_{x}^{2} / 2 m_{e}^{*}+\left(p_{y}+e B x\right)^{2} / 2 m_{e}^{*}+p_{z}^{2} / 2 m_{e}^{*}+V_{\text {conf }}(z) \\
& +J_{s-d} \sum_{i} \mathbf{S}(\mathbf{r}) \cdot \mathbf{S}\left(\mathbf{R}_{i}\right) \delta\left(\mathbf{r}-\mathbf{R}_{i}\right),
\end{aligned}
$$

where $V_{\text {conf }}(z)=V_{\text {conf }}(z+L)$ is the periodic potential along the growth direction, $L$ is the period of the DMS superlattice, $\mathbf{S}$ is the spin of the localized $3 d^{5}$ electrons of the $\mathrm{Mn}$ ions with $S=5 / 2$, and $\mathbf{s}$ is the electron spin. We assume that the magnetic ions are distributed homogeneously in the DMS layers. The extended nature of the electronic wave function spanning a large number of magnetic ions and lattice sites allows the use of the molecular-field approximation to replace the magnetic-ion spin operator $\mathbf{S}_{i}$ with its thermal and spatial average $\left\langle S_{z}\right\rangle$, taken over all the ions. The mean spin $\left\langle S_{z}\right\rangle$ denotes the spatial as well as the thermal average of the spin component along the magnetic field. This approach has been proven to be valid in previous theoretical works on the study of the magneto-optical properties of DMS material. Very recently, this approximation was applied successfully to study the transport property of a DMS system. ${ }^{16,19}$ The exchange interaction given by Eq. (1) can, in the molecularfield approximation, be written in terms of a Zeeman-like Hamiltonian

$$
\begin{aligned}
H= & p_{x}^{2} / 2 m_{e}^{*}+\left(p_{y}+e B x\right)^{2} / 2 m_{e}^{*} \\
& +p_{z}^{2} / 2 m_{e}^{*}+V_{\text {conf }}(z)+J_{s-d}\left\langle S_{z}\right\rangle \sigma_{z} \\
= & p_{x}^{2} / 2 m_{e}^{*}+\left(p_{y}+e B x\right)^{2} / 2 m_{e}^{*}+p_{z}^{2} / 2 m_{e}^{*}+V_{e f f}(z),
\end{aligned}
$$

where $\quad J_{s-d}=-N_{0} \alpha x$ and $\left\langle S_{z}\right\rangle=5 / 2 B_{J}\left(S g \mu_{B} B / k_{B}(T\right.$ $\left.\left.+T_{0}\right)\right), B_{J}(x)$ is the Brillouin function, $N_{0}$ is the number of cations per unit volume, $x$ is the $\mathrm{Mn}$ concentration, $T_{0}$ accounts for the reduced single-ion contribution due to the antiferromagnetic Mn-Mn coupling, $k_{B}$ is the Boltzmann constant, and $\sigma$ is the electron spin. The parameters used in the calculation are taken from Ref. 3: $m_{e}^{*}=0.16 m_{0}, x=0.04$, $V_{\text {conf }}=-3 \mathrm{meV}$ in the DMS material, $g=2, N_{0} \alpha$ $=0.27 \mathrm{eV}, T_{0}=1.4 \mathrm{~K}$. In Eq. (2) the exchange interaction $H_{s-d}=J_{s-d}\left\langle S_{z}\right\rangle s_{z}$ only induces spin-conserving processes, and consequently we neglected all spin-flip processes.

Using the usual boundary conditions ${ }^{20}$ for the electron wave function at the well/barrier interface, the energy eigenvalue can be obtained by solving the following equation:

$$
\begin{aligned}
\cos k_{z} L= & \cos k_{N}^{s} L_{N} \cos k_{D}^{s} L_{D}-\frac{1}{2}\left(\frac{m_{D} k_{N}}{m_{N} k_{D}}\right. \\
& \left.+\frac{m_{N} k_{D}}{m_{W} k_{N}}\right) \sin k_{N}^{s} L_{N} \sin k_{D}^{s} L_{D},
\end{aligned}
$$

where $k_{N}^{s}=\sqrt{2 m_{N}\left(E-V_{N}^{s}\right) / \hbar^{2}}, k_{D}^{s}=\sqrt{2 m_{D}\left(E-V_{D}^{s}\right) / \hbar^{2}}$, and $V_{N}^{S}=0, \quad V_{D}^{S}=V_{\text {conf }} \pm 1 / 2 N_{0} \alpha x\left\langle S_{z}\right\rangle$ is the depth or the height of the DMS layer that depends on the spin orientation ( $s= \pm$, " + " for the spin-up electron, " - " for the spindown electron). The period of the superlattice is $L=L_{D}$ $+L_{N}$, where $L_{D}$ is the width of the DMS layers and $L_{N}$ is the width of the nonmagnetic semiconductor layers. $m_{D}$ and $m_{N}$ denote the effective mass of the electron in the DMS layers and nonmagnetic semiconductor layers, respectively. In this work the difference between the effective mass of the electron in the DMS layers and the non-DMS layers is neglected that is a reasonable assumption because of the low Mn concentration $\left(m_{N}=m_{D}=m_{e}^{*}\right)$. Notice that the barrier 
height and the well depth can be tuned by varying the external magnetic field. The wave function of the electron for spin $s$ in the DMS superlattice can be expressed as

$$
\Psi=\frac{1}{\sqrt{L_{y}}} \phi_{n}\left(x-X_{k_{y}}\right) e^{i k_{y} y} \psi_{k_{z}}(z),
$$

where $X_{k_{y}}=-k_{y} l_{B}^{2}, l_{B}=\sqrt{\hbar / e B}$ is the magnetic length, $\psi_{k_{z}}(z)=1 / \sqrt{L_{z}} e^{i k_{z} z} u_{k_{z} s}(z)$ is the envelope function along the $z$ axis, $\phi_{n}(x)$ is the wave function of the harmonic oscillator,

$$
\phi_{n}\left(x-X_{k_{y}}\right)=\frac{1}{\pi^{1 / 4} \sqrt{2^{n} n ! l_{B}}} \exp \left[-\frac{\left(x-X_{k_{y}}\right)^{2}}{2 l_{B}^{2}}\right] H_{n}\left[\frac{x-X_{k_{y}}}{l_{B}}\right],
$$

where $H_{n}(x)$ is the $n$th Hermite polynomial.

In the presence of a magnetic field, the in-plane motion of the electron is described by discrete Landau levels on which any effect of the collision broadening is ignored. Therefore, the eigenvalue of the electron state in a DMS superlattice under a perpendicular magnetic field is

$$
\mathcal{E}(\widetilde{k})=\left(n+\frac{1}{2}\right) \hbar \omega_{c}+\mathcal{E}_{s}\left(k_{z}\right),
$$

where $\widetilde{k}=\left(n, k_{z}, s\right)$ is the complete set of quantum indices, $\omega_{c}=e B / m_{e}^{*}$ is the cyclotron frequency, and $n$ denotes the label for the Landau level. $\mathcal{E}_{s}\left(k_{z}\right)$ is the energy spectrum of the miniband in the DMS superlattice.

The group velocity of the electron along the $z$ direction is

$$
v_{s, z}=\frac{1}{\hbar} \frac{\partial \mathcal{E}\left(k_{z}\right)}{\partial k_{z}}, \quad s=\uparrow, \downarrow .
$$

The ballistic current density $J^{s}$ is the sum of the contributions from each Landau level with different spin

$$
J=-n e\left\langle v_{z}\right\rangle=-\frac{e E}{2 \pi l_{B}^{2}} \sum_{s, n} \int_{-\pi / L}^{\pi / L} \frac{d k_{z}}{2 \pi} v_{s, z}\left(n, k_{z}\right) f_{s}\left(n, k_{z}\right),
$$

where $1 / 2 \pi l_{B}^{2}$ is the degeneracy of the Landau level for each spin, $f_{s}\left(n, k_{z}\right)$ is the electron distribution function in the state $\left(n, k_{z}, s\right)$ that can be determined from the semiclassical Boltzmann equation

$$
\frac{\partial f_{s}}{\partial t}+\mathbf{v} \cdot \boldsymbol{\nabla} f_{s}+\frac{\partial \mathbf{k}}{\partial t} \cdot \nabla_{k} f_{s}=\left(\frac{\partial f_{0}}{\partial t}\right)_{c} .
$$

If the distribution $f_{s}$ depends weakly on the position $z$ along the growth direction, and is independent of the time, Eq. (9) becomes

$$
-e \mathbf{E} \cdot \nabla_{k} f_{s}=\left(\frac{\partial f_{0}}{\partial t}\right)_{c} .
$$

If we use the relaxation-time approximation, the collision term on the right side of Eq. (10) is equal to

$$
\left(\frac{\partial f_{0}}{\partial t}\right)_{c}=-\frac{f(\widetilde{k})-f_{0}(\widetilde{k})}{\tau(\widetilde{k})},
$$

where $f_{0}(\mathcal{E}(\widetilde{k}))=1 /\left\{\exp \left[\left(\mathcal{E}(\widetilde{k})-E_{F}\right] / k_{B} T\right)+1\right\}$ is the FermiDirac distribution, $E$ is the electric field along the growth direction, and $\tau(\widetilde{k})$ denotes the electron relaxation time in the DMS superlattice.

The Fermi energy can be determined from the following equation:

$$
n_{e}=\frac{e^{2}}{4 \pi^{2} l_{B}^{2}} \sum_{s, n} \int_{-\pi / L}^{\pi / L} f\left(\mathcal{E}\left(n, k_{z}, s\right)\right) d k_{z},
$$

where $n_{e}$ is the density of the electron in the DMS superlattice.

We restrict ourselves to the linear-response regime, assume weak electric field, and ignore spin-flip processes, therefore, the distribution function can be written in the form of $f=f_{0}+f_{1}=f_{0}-e v \tau E \partial f_{0} / \partial \mathcal{E}$, here $f_{0}$ is the equilibrium distribution function and $f_{1}$ is the linear term that is proportional to the electric field. Because there is no electric current in the equilibrium Fermi-Dirac distribution, the current density Eq. (8) becomes

$$
J=\frac{e^{2} E \tau}{4 \pi^{2} l_{B}^{2}} \sum_{s, n} \int_{-\pi / L}^{\pi / L} d k_{z}\left(-\frac{\partial f_{0}}{\partial \mathcal{E}}\right) v_{s, z}^{2} .
$$

From this formula, we can find that the current density is ascribed to the contribution of the Landau level near the Fermi energy, and especially at low temperature $-\left(\partial f_{0} / \partial \mathcal{E}\right) \approx \delta\left(\mathcal{E}-\mathcal{E}_{F}\right)$ for $k_{B} T \ll \mathcal{E}_{F}$. The conductivity $\sigma$ can be obtained as

$$
\sigma=\frac{e^{2} \tau}{4 \pi^{2} l_{B}^{2}} \sum_{s, n} \int_{-\pi / L}^{\pi / L} d k_{z}\left(-\frac{\partial f_{0}}{\partial \mathcal{E}}\right) v_{s, z}^{2} .
$$

The degree of spin polarization of the current density under weak electric field can be defined as

$$
P=\frac{J^{\downarrow}-J^{\uparrow}}{J^{\downarrow}+J^{\uparrow}},
$$

here $J^{\uparrow}\left(J^{\downarrow}\right)$ is the component of spin-up (spin-down) current density.

For simplicity, we take the relaxation time $\tau$ as a constant in the calculation of the conductivity. In the DMS system, the relaxation time $\tau$ takes into account energy- and spinrelaxation processes and may be written in the form

$$
\frac{1}{\tau}=\frac{1}{\tau_{e}}+\frac{1}{\tau_{s-d}} .
$$

Here, $\tau_{e}$ denotes the relaxation time that incorporates other energy and momentum scattering mechanism and $\tau_{s-d}$ denotes spin-related scattering processes that is caused by the $s-d$ interaction. 
In order to estimate the effect of the magnetization fluctuation, we calculate the relaxation time $\tau_{s-d}$, i.e., the scattering process induced by the $s-d$ exchange interaction between the conduction electron and the magnetic ions. The transition rate from an initial state $\left|n, k_{z}, \sigma_{z}, S_{1}^{z}, S_{1}^{z}, \ldots, S_{N}^{z}\right\rangle$ to all the final states $\left|n^{\prime}, k_{z}^{\prime}, \sigma_{z}^{\prime}, S_{1}^{\prime z}, S_{1}^{\prime z}, \ldots, S_{N}^{\prime z}\right\rangle$ can be calculated using the Fermi golden rule and after performing an ensemble average over a distribution of the magnetic ions

$$
W_{\widetilde{k} \widetilde{k}^{\prime}}=\frac{2 \pi}{\hbar}\left|\left\langle\widetilde{k}\left|V_{s-d}\right| \widetilde{k}^{\prime}\right\rangle\right|^{2},
$$

where $V_{s-d}=J_{s-d} \Sigma_{i} \mathbf{S}(\mathbf{r}) \cdot \mathbf{S}\left(\mathbf{R}_{i}\right) \delta\left(\mathbf{r}-\mathbf{R}_{i}\right)$ describes the $s-d$ exchange interaction between the conduction electron and the magnetic ions, $\mathbf{R}_{i}$ is the coordinate of the magnetic ion $\mathrm{Mn}^{2+}$. The matrix element of the $s-d$ interaction can be obtained $^{14,23}$

$$
\begin{aligned}
\overline{\left|\left\langle\widetilde{k}\left|V_{s-d}\right| \widetilde{k}^{\prime}\right\rangle\right|^{2}}= & J_{s-d}^{2} \sum_{i, j} \frac{1}{L_{z}^{2}} \exp \left[i\left(k_{z}-k_{z}^{\prime}\right)\left(Z_{i}-Z_{j}\right)\right]\left\{\left\langle S_{i}^{z} S_{j}^{z}\right\rangle \delta_{\sigma \sigma^{\prime}}+\left\langle S_{i}^{\mp} S_{j}^{ \pm}\right\rangle\left(1-\delta_{\sigma \sigma^{\prime}}\right)\right\} \sum_{n^{\prime}, k_{z}^{\prime}} \delta\left(\mathcal{E}\left(n, k_{z}, \sigma\right)\right. \\
& \left.-\mathcal{E}\left(n^{\prime}, k_{z}^{\prime}, \sigma^{\prime}\right)\right) \frac{1}{\Omega^{N}} \int\left(\prod_{i} d \mathbf{R}_{i}\right) \phi_{n}^{*}\left(X_{i}\right) \phi_{n}\left(X_{i}\right) \phi_{n^{\prime}}^{*}\left(X_{j}\right) \phi_{n^{\prime}}\left(X_{j}\right) u_{k_{z}}^{*}\left(Z_{i}\right) u_{k_{z}^{\prime}}\left(Z_{i}\right) u_{k_{z}}^{*}\left(Z_{j}\right) u_{k_{z}^{\prime}}\left(Z_{j}\right),
\end{aligned}
$$

where the capital letters denote the coordinates of the magnetic ions, $S_{i}^{ \pm}=S_{i}^{x} \pm i S_{i}^{y}, \delta_{\sigma \sigma^{\prime}}=1$ for $\sigma=\sigma^{\prime}$, and 0 otherwise. $\Omega$ is the volume of the sample, $N$ the number of the magnetic ions. The brackets $\langle\cdots\rangle$ indicate the statistical average over $\mathrm{Mn}$ spin orientation $\mid S_{z}>\left(S_{z}=-5 / 2\right.$, $-3 / 2, \ldots, 3 / 2,5 / 2)$. The term $\left\langle S_{i}^{z} S_{j}^{z}\right\rangle$ in Eq. (18) describes the spin-conserved scattering process and the term $\left\langle S_{i}^{\mp} S_{j}^{ \pm}\right\rangle$ indicates the spin-flip scattering process. ${ }^{23}$ Neglecting the terms where $i \neq j$ in Eq. (18) that do not contribute to the change of the distribution function, then we obtain

$$
\begin{aligned}
\overline{\left|\left\langle\widetilde{k}\left|V_{s-d}\right| \tilde{k}^{\prime}\right\rangle\right|^{2}}= & J_{s-d}^{2} \sum_{i}\left\{\left\langle S_{i}^{z} S_{i}^{z}\right\rangle \delta_{\sigma \sigma^{\prime}}+\left\langle S_{i}^{\mp} S_{i}^{ \pm}\right\rangle\left(1-\delta_{\sigma \sigma^{\prime}}\right)\right\} \\
& \times \sum_{n^{\prime}, k_{z}^{\prime}} \delta\left(\mathcal{E}\left(n, k_{z}, \sigma\right)-\mathcal{E}\left(n^{\prime}, k_{z}^{\prime}, \sigma\right)\right) \\
& \times \frac{\Omega}{N L_{y}} \int\left|\phi_{n}\left(X_{i}\right)\right|^{2}\left|\phi_{n^{\prime}}\left(X_{i}\right)\right|^{2} I_{k k^{\prime}} d X, \quad \text { (19) }
\end{aligned}
$$

where $I_{k k^{\prime}}=\int\left|\psi_{k_{z}}\left(z_{i}\right)\right|^{2}\left|\psi_{k_{z}^{\prime}}\left(z_{i}\right)\right|^{2} d z_{i}$. The relaxation time $\tau_{s-d}$ due to the $s-d$ interaction can be obtained after a sum over all possible final states,

$$
\begin{aligned}
\frac{1}{\tau_{s-d}(\widetilde{k})}= & \frac{2 \pi}{\hbar} \sum_{n^{\prime}} \frac{1}{\left|v\left(\widetilde{k}^{\prime}\right)\right|} \frac{L_{y}}{2 \pi} \int \overline{\left|\left\langle\widetilde{k}\left|V_{s-d}\right| \widetilde{k}^{\prime}\right\rangle\right|^{2}} d k_{y}^{\prime} \\
= & \frac{2 L_{z} J_{s-d}^{2}}{\pi \hbar^{2} l_{B}^{2}} \frac{\Omega}{N}\left\{\left\langle\left(S^{z}\right)^{2}\right\rangle \delta_{\sigma \sigma^{\prime}}+\left\langle S^{\mp} S^{ \pm}\right\rangle\left(1-\delta_{\sigma \sigma^{\prime}}\right)\right\} \\
& \times \sum_{n^{\prime}} \frac{I_{k k^{\prime}}}{\left|v\left(\widetilde{k}^{\prime}\right)\right|} \delta\left(\mathcal{E}\left(n, k_{z}, \sigma\right)-\mathcal{E}\left(n^{\prime}, k_{z}^{\prime}, \sigma^{\prime}\right)\right),
\end{aligned}
$$

where $v\left(\widetilde{k}^{\prime}\right)$ is the electron group velocity of the final states along the growth direction of the superlattice.

\section{NUMERICAL RESULTS AND DISCUSSIONS}

Figures 2(a) and 2(b) show the energy spectrum of the lowest two miniband spin-up (solid curves) and spin-down (dashed curves) electron states [see Eq. (6), $n=0$ ] for a $\mathrm{ZnSe} / \mathrm{Zn}_{0.96} \mathrm{Mn}_{0.04} \mathrm{Se}$ diluted magnetic semiconductor superlattice for different magnetic fields. For definition, the width of the barrier (well) is taken as $L_{D}=10 \mathrm{~nm}\left(L_{N}=10 \mathrm{~nm}\right)$ throughout the paper. From these figures we notice that the separation between the spin-up and spin-down electron is enhanced significantly with increasing magnetic field. Notice that the Fermi energy is located slightly above the bottom of the second miniband at low magnetic fields [see Fig. 2(a)]. In strong magnetic fields, the energy of the lowest spin-up miniband is even higher than that of the spin-down second miniband, and only the lowest spin-down miniband is occupied by electrons [see Fig. 2(b)]. This can be explained as follows, an external magnetic field induces a magnetization of the magnetic ion $\mathrm{Mn}^{2+}$ along the direction of the magnetic field in the DMS superlattice. From Eq. (1), the magnetic ions can influence the energy of the electron state via the $s$ - $d$ exchange interaction, and leads to a giant spin splitting that is comparable to the band offset between $\mathrm{ZnSe}$ and $\mathrm{Zn}_{0.96} \mathrm{Mn}_{0.04} \mathrm{Se}$.

Figures 2(c) and 2(d) depict how the bandwidths of the electrons for different spin orientation vary with the magnetic field. For spin-down electrons the bandwidth decreases and saturates with increasing magnetic field, but for spin-up electrons, it exhibits a maximum and saturates when the magnetic field increases. For spin-up electrons the wells become more and more shallow and finally form barriers with increasing magnetic field. At this point the bandwidths diverge and the miniband gaps disappear [see Fig. 2(c)]. Therefore the bandwidth for the spin-up electron exhibits a local maximum. In Fig. 2(e) we plot the group velocity of the electrons with different spin orientation as a function of the momentum $k_{z}$ for different magnetic fields. At low fields the group velocity for the spin-up electron is larger than that in the absence of magnetic field. The group velocity for the 

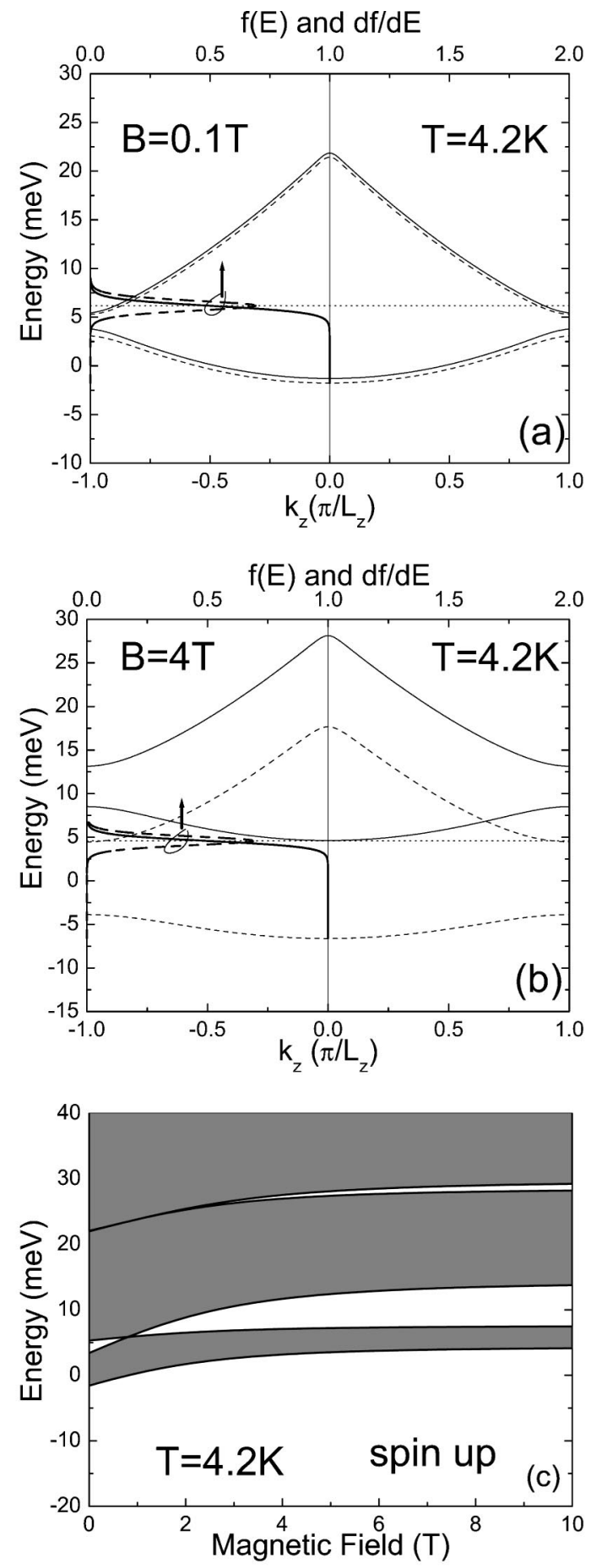
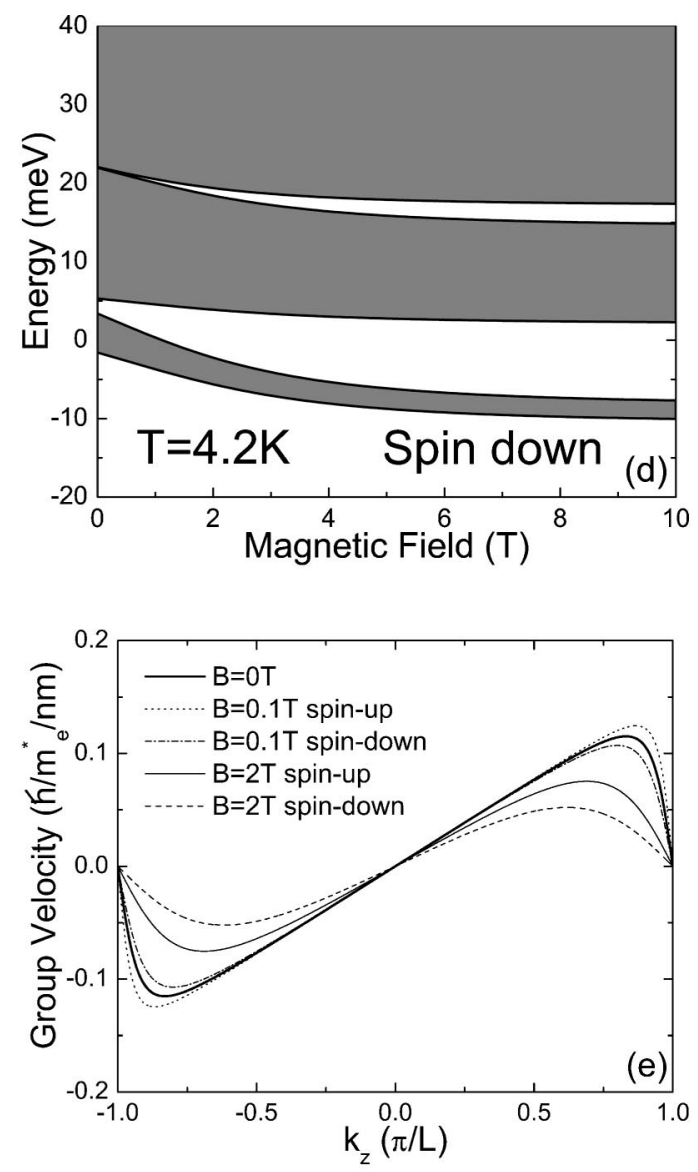

FIG. 2. The energy spectrum of the two lowest minibands of electron states $(n=0)$ in a DMS superlattice for different spin orientations under two different magnetic fields: (a) $\mathrm{B}=0.1 \mathrm{~T}$ and (b) $\mathrm{B}=4 \mathrm{~T}$. The width of the barrier (well) is $L_{D}=10 \mathrm{~nm}\left(L_{N}=10 \mathrm{~nm}\right)$, the lattice period $L=L_{D}+L_{N}$ and $T=4.2 \mathrm{~K}$. The density of the electrons is $n_{e}=2 \times 10^{17} / \mathrm{cm}^{3}$. The solid curves denote the energy spectrum for the spin-up electron, the dashed curves for the spin-down electron, the dotted line is the Fermi energy, the thick solid and dashed curve denote the derivative of the Fermi distribution and the Fermi distribution, $\partial f / \partial \mathcal{E}$ and $f(\mathcal{E})$, near the Fermi energy, respectively. The bandwidth of the two lowest spin-up (c) and spin-down (d) bands are shown as function of the magnetic field. The shaded regions in the figures denote the electron miniband $\left[\mathcal{E}_{s}\left(k_{z}\right)\right]$ in the DMS superlattice. (e) shows the group velocity of the spin-up and spin-down electron lowest miniband as a function of the momentum $k_{z}$ for different magnetic fields.

spin-up and spin-down electrons decrease at strong magnetic fields.

Figure 3 shows the Landau-level fan diagram for spindown (thick curves) and spin-up (thin curves) electrons in a
DMS superlattice under a perpendicular magnetic field. The solid (dashed) lines indicate the energy of the Landau level at $k_{z}=0 \quad\left(k_{z}=\pi / L_{z}\right)$, i.e., the edge of the lowest miniband. Notice that the magnetic-field variation of the Landau levels 


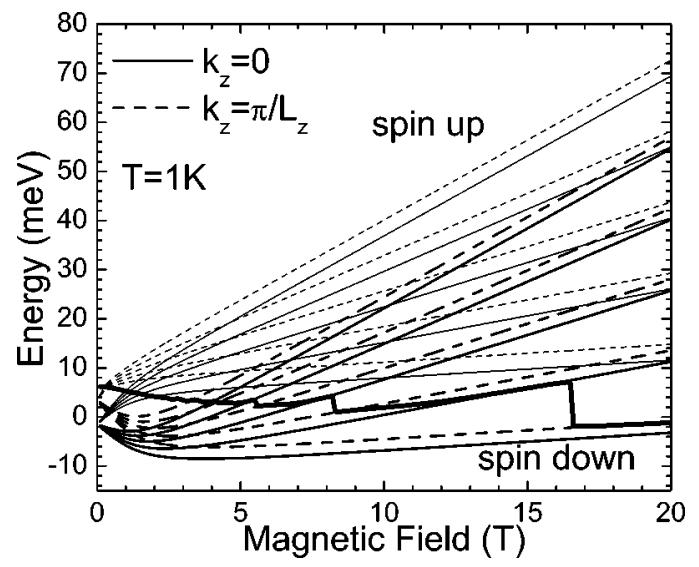

FIG. 3. The energy spectrum of the lowest miniband for different Landau levels for spin-up (thin curves) and spin-down (thick curves) electrons as a function of magnetic field. The solid lines and dashed lines represent the energy of electrons at $k_{z}=0$ and at $k_{z}=\pi / L$, respectively. The thick solid and thick dashed lines show the energies for the spin-down electron at $k_{z}=0$. The thickest solid curve shows the Fermi energy as a function of magnetic field. $L_{D}=10 \mathrm{~nm}, L_{N}=10 \mathrm{~nm}$, the density of the electrons is $n_{e}=2 \times 10^{17} / \mathrm{cm}^{3}$.

in DMS is quite different from that in a nonmagnetic semiconductor, which is linearly proportional to the external magnetic field. In a DMS superlattice the Landau levels of the spin-down electron exhibit minima with increasing magnetic field, and its variation with magnetic field is quite different from that of the Landau levels for the spin-up electron. For very small magnetic fields the $s-d$ exchange interaction increases the barrier height for the spin-up electrons moving the Landau levels up in energy, while for the spin-down electrons the wells deepen resulting in a decrease of the Landaulevel energy. This effect saturates around $B \sim 4 \mathrm{~T}$ when the magnetization of the $\mathrm{Mn}^{2+}$ is saturated beyond which we have the usual Landau-level fan diagram for each of the electron-spin states. The spin-up and spin-down fan are shifted in energy due to the fact that they move in a different superlattice potential. The thickest solid curve in Fig. 3 denotes the Fermi energy vs the magnetic field. Sharp drops take place at the points where the Fermi energy passes through the bottom of the different Landau-level bands. From this figure we also learn that the electron state becomes spin polarized since only the lowest spin-down miniband is populated at sufficient large fields.

Figure 4 shows the conductivity $\sigma$ as a function of magnetic field for various temperatures in a DMS superlattice. The inset shows the Fermi energy vs magnetic field in such a system. An interesting property of the MC is the variation of the low-field MC. The conductivity decreases and oscillates with increasing magnetic field. At low magnetic field, the spin splitting induced by the $s-d$ exchange interaction is even much larger than the separation of the Landau levels, the $s-d$ exchange interaction results in a variation of the miniband width, i.e., a variation of the electron group velocity [see Eq. (7)]. From Figs. 2(c) and 2(d), we find that an increase of the magnetic field leads to a decrease of the bandwidth for the spin-down and the spin-up electron and a local maximum for

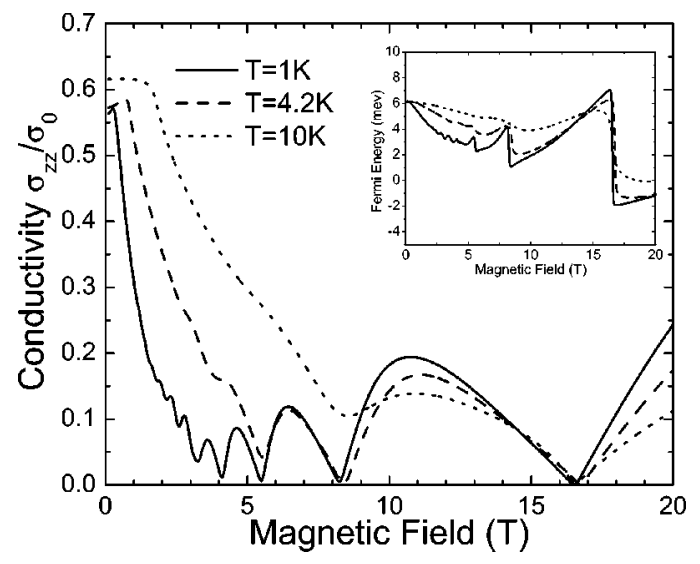

FIG. 4. The conductivity $\sigma_{z z} / \sigma_{0}$ vs the magnetic field for three different electron temperatures, where $\sigma_{0}=n_{e} e^{2} \tau / m^{*} . L_{D}$ $=10 \mathrm{~nm}, L_{N}=10 \mathrm{~nm}$, and the density of the electrons $n_{e}=2$ $\times 10^{17} / \mathrm{cm}^{3}$. The inset shows the Fermi energy as a function of magnetic field.

the spin-up electron, i.e., a decrease of the group velocity for the spin-down and spin-up electron and a local maximum for the spin-up electron [see Fig. 2(e)]. Therefore, the MC exhibits a maximum for the spin-up electron and a decrease for the spin-down electron in the low-field case (see Fig. 4). The decrease of the low-field MC was found previously in disordered two-dimensional system that was attributed to quantum corrections caused by Anderson weak localization. ${ }^{22}$ But the decrease of MC in a DMS superlattice arises from the $s-d$ exchange interaction between the electron and the localized magnetic impurity that lifts the degeneracy of the spin-up and spin-down electron band states.

The magnetization of the magnetic ions $\mathrm{Mn}^{2+}$ saturates with increasing magnetic field, therefore the strength of the exchange interaction [the last term in Eq. (2)] also saturates when the magnetic field becomes strong enough $(B>4 \mathrm{~T})$. The separation of the Landau levels increases linearly (see Fig. 3) with increasing magnetic field. The Fermi surface passes through the band bottom of the subsequent Landau levels with increasing magnetic field. The Fermi energy (see the inset) decreases and shows a series of sharp drops at strong magnetic field. The variation of $\mathrm{MC}$ in strong magnetic field is ascribed to the contribution from Landau levels near the Fermi energy. When a Landau level passes through the Fermi surface, the electron group velocity of the states that contribute to conduction drops to zero [see Eq. (14)] resulting in an oscillation of the MC. The conductivity exhibits a sharp dip if there is only one Landau level near the Fermi energy. The separation of the Landau levels is small at low field and these dips are smeared out since there are many Landau levels located near the Fermi surface. From this figure, we can also see that the dips will be less pronounced when temperature increases since the latter leads to a smearing of the Fermi surface.

Figure 5 depicts how the conductivity $\sigma$ varies with magnetic field for different carrier density. The period of the conductivity oscillations for lower density is larger than that for higher density, which can be understood from the inset which shows the variation of the Fermi energy vs magnetic 


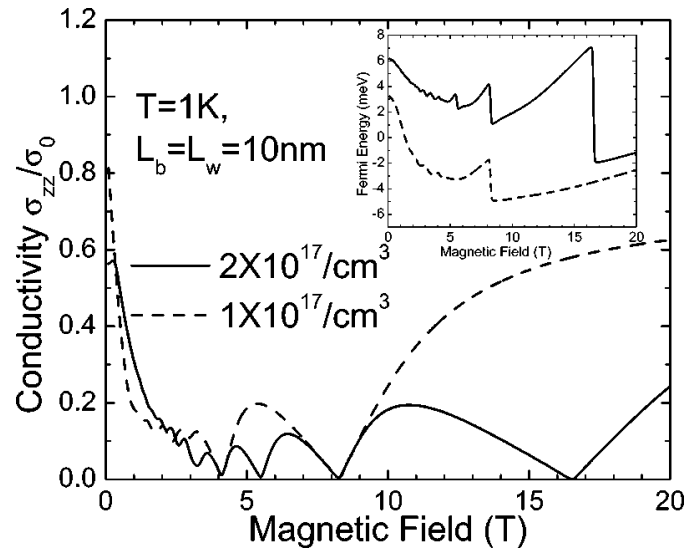

FIG. 5. The conductivity $\sigma_{z z} / \sigma_{0}$ vs the magnetic field for two different electron densities. $L_{D}=10 \mathrm{~nm}, L_{N}=10 \mathrm{~nm}, T=1 \mathrm{~K}$. The inset shows the Fermi energy for two different densities.

field. When the Landau level passes through the Fermi surface, a corresponding dip can be found in the conductivity. Since the period of the oscillation of the Fermi energy for lower density is also larger than that for higher density, the period of the MC oscillation for lower density will be larger than for higher density.

In Fig. 6 we plot the spin polarization of the current vs the magnetic field for different temperatures. The inset shows the spin-up and spin-down components of MC. The spin-up components exhibit a maximum for small magnetic field and decreases rapidly to zero, since the population for the spin-up band decreases when the magnetic field increases. The maximum is due to a maximum in the bandwidth [see Fig. 2(c)]. From this inset we found that the oscillation in the $\mathrm{MC}$ is due to the spin-down MC components. The spin polarization at higher temperature increases more slowly than that at low temperature due to the thermal fluctuations of the magnetization of the magnetic ions.

In order to estimate the effect of the spin-disorder scattering process on the total relaxation time $\tau$, we show the re-

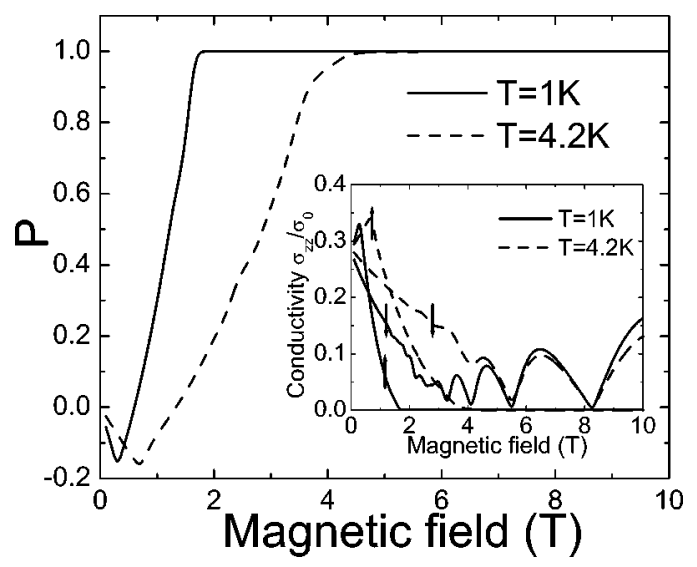

FIG. 6. The spin polarization of the current in a DMS superlattice for different temperatures. The inset shows the spin-up and spin-down MC components as a function of magnetic field. The arrows in the inset represent the spin-up and spin-down MC components. $L_{D}=10 \mathrm{~nm}, L_{N}=10 \mathrm{~nm}, T=1 \mathrm{~K}$.

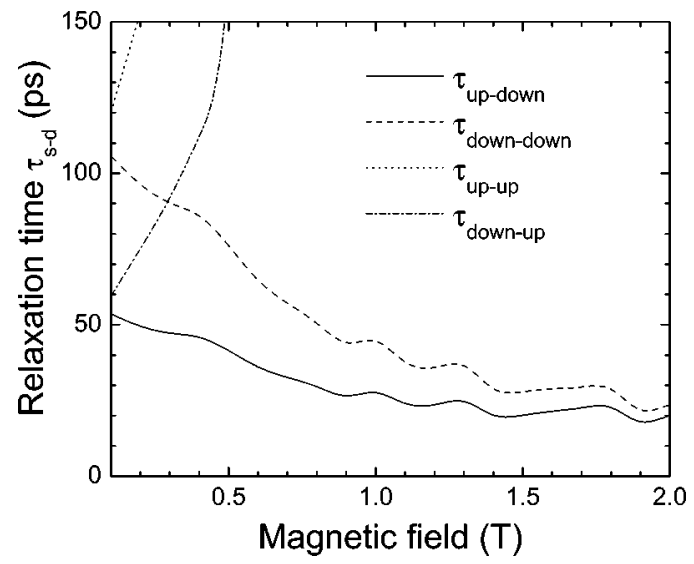

FIG. 7. The relaxation times due to the spin-disorder scattering processes vs the magnetic field at $T=4.2 \mathrm{~K} . \tau_{u p-u p}\left(\tau_{u p-d o w n}\right)$ denotes the relaxation time for the transition in which the initial state is the spin-up Landau level near the Fermi energy and the final states are the spin-up (spin-down) Landau levels. The definition of the relaxation time $\tau_{\text {down-down }}\left(\tau_{\text {down-up }}\right)$ is similar.

laxation time induced by the $s-d$ exchange interaction as a function of the external magnetic field in Fig. 7. Since only electrons near the Fermi surface contribute to the conductivity, therefore, we calculate the relaxation time of the highest occupied Landau levels. Notice that our definition of the relaxation time follows the one of Ref. 14 which is similar to Eq. (25) of Ref. 23. From these numerical results we find that the spin-conserved and the spin-flip relaxation time are rather long as compared to the relaxation time due to the other energy and momentum relaxation processes, such as the electron-optical phonon scattering, the electron-acoustic phonon scattering, and the electron-impurity scattering processes, etc. A rough estimate of the relaxation time $\tau_{e}$ from PL experiments give $\left(\tau_{e} \sim 3-4 \mathrm{ps}\right) .{ }^{15}$ An approximate estimate from the mobility of the $\mathrm{ZnSe}$ semiconductor material gives $\left(\tau_{e} \sim 1-10 \mathrm{ps}\right) .^{21}$ As a consequence we see from Eq. (16) that the total relaxation time is mainly determined by the non-spin-disorder scattering mechanisms $\tau_{e}$, hence we may neglect $\tau_{s-d}$ for practical purposes.

\section{CONCLUSIONS}

We studied the electron transport in DMS superlattices using a semiclassical Boltzmann equation, and investigated the effect of the $s-d$ exchange interaction, which is treated using the molecular-field approximation, on the longitudinal spin transport in diluted magnetic semiconductor superlattices. The conductivity exhibits an oscillating behavior with varying magnetic field. The conductivity decreases rapidly for small magnetic field, and increases for strong magnetic field. The dips in the conductivity at strong magnetic fields are smeared out with increasing temperature. The spin polarization increases rapidly with increasing magnetic field and the longitudinal current becomes spin polarized in strong magnetic fields. Our results clearly illustrate that one can adjust the longitudinal spin transport by tuning the external magnetic field in DMS superlattices. Most optical and transport properties of the band electrons were successfully inter- 
preted within the molecular-field approximation for pure paramagnetic DMS. This approach is only justified for pure paramagnetic material where every spin can be treated independently.

From our numerical results, it is reasonable to ignore the spin-disorder scattering process, i.e., the effect of the magnetization fluctuations, which is less important than other scattering processes in this DMS superlattice structure. In summary, the external magnetic field provides us a tool to tailor the transport properties of DMS superlattices. Such systems are extremely attractive from the point of view of both basic research and technological application, such as for a spin filter.

\section{ACKNOWLEDGMENTS}

This work was supported by the foundation from the Chinese Academy of Sciences, the Chinese National Science Foundation, the Flemish Science Foundation (FWO-Vl), the Interuniversity Microelectronics Center (IMEC), the InterUniversity Attraction Poles (IUAP) research program, the Flemish Concerted Action program (GOA), and the FlemishChina bilateral science and technological cooperation.
*Electronic address: kchang@red.semi.ac.cn

†Electronic address: peeters@uia.ua.ac.be

${ }^{1}$ Diluted Magnetic Semiconductors, edited by J. K. Furdyna and J. Kossut, Semiconductors and Semimetals Vol. 25 (Academic, New York, 1988).

${ }^{2}$ N. Dai, H. Luo, F. C. Zhang, N. Sarmarth, M. Dobrowolska, and J. K. Furdyna, Phys. Rev. Lett. 67, 3824 (1991).

${ }^{3}$ N. Dai, L. R. Ram-Mohan, H. Luo, G. L. Yang, F. C. Zhang, M. Dobrowolska, and J. K. Furdyna, Phys. Rev. B 50, 18153 (1994).

${ }^{4}$ M. Oestreich, J. Hubner, D. Hagele, P. J. Kar, W. Heimbrodt, W. W. Ruhle, D. E. Ashenford, and B. Lunn, Appl. Phys. Lett. 74, 1251 (1999).

${ }^{5}$ J. Kossut, Phys. Status Solidi B 72, 359 (1975).

${ }^{6}$ J. K. Furdyna, J. Appl. Phys. 64, R29 (1988).

${ }^{7}$ M. von Ortenberg, Phys. Rev. Lett. 49, 1041 (1982).

${ }^{8}$ G. A. Prinz, Phys. Today 48(4), 58 (1995); G. A. Prinz, Science 282, 1660 (1998).

${ }^{9}$ J. M. Kikkawa, I. P. Smorchkova, N. Samarth, and D. D. Awshalom, Science 277, 1284 (1997); J. M. Kikkawa and D. D. Awshalom, Nature (London) 397, 139 (1999).

${ }^{10}$ R. Fiederling, M. Keim, G. Reuscher, W. Ossau, G. Schmidt, A. Waag, and L. W. Molenkamp, Nature (London) 402, 787 (1999).

${ }^{11}$ Y. Ohno, D. K. Young, B. Benschoten, F. Matsukura, H. Ohno, and D. D. Awschalom, Nature (London) 402, 790 (1999).

${ }^{12}$ J. Hajdu and G. Landwehr, in Strong and Ultrastrong Magnetic Fields and Their Applications, edited by F. Herlach (Springer-
Verlag, New York, 1985), pp. 17-112.

${ }^{13}$ V. M. Polyanovskii, Sov. Phys. Semicond. 18, 1142 (1984).

${ }^{14}$ A. E. Datars and J. E. Sipe, Phys. Rev. B 51, 4312 (1995).

${ }^{15}$ G. Bastard and R. Ferreira, Surf. Sci. 267, 335 (1992); G. Bastard and L. L. Chang, Phys. Rev. B 41, 7899 (1990).

${ }^{16}$ A. Lemaitre, C. Testelin, C. Rigaux, T. Wojtowicz, and G. Karczewski, Phys. Rev. B 62, 5059 (2000).

${ }^{17}$ V. V. Rossin, F. Henneberger, and J. Pulsm, Phys. Rev. B 53, 16444 (1996).

${ }^{18}$ S. Lee, M. Dobrowolska, J. K. Furdyna, H. Luo, and L. R. RamMahan, Phys. Rev. B 54, 16939 (1996); S. Lee, M. Dobrowolska, J. K. Furdyna, and L. R. Ram-Mohan, ibid. 59, 10302 (1999).

${ }^{19}$ J. C. Egues, Phys. Rev. Lett. 80, 4578 (1998); K. Chang and F.M. Peeters, Solid State Commun. 120, 181 (2001).

${ }^{20}$ The formulism used in our calculation can be found in the work of F. Szmulowicz, Phys. Rev. B 54, 11539 (1996), which is based on the exact envelope-function formulism developed by M. G. Burt, J. Phys.: Condens. Matter 4, 6651 (1992). In the case of a one-band model, it reduced to the familiar KronigPenney model result [G. Bastard, Phys. Rev. B 25, 7584 (1982)].

${ }^{21}$ O. Madelung, M. Schultz, and H. Weiss, Numerical Data and Functional Relationships in Science and Technology (SpringerVerlag, Berlin, 1982), Vol. 17, p. 146.

${ }^{22}$ P. A. Lee and T. V. Ramakrishnan, Rev. Mod. Phys. 57, 287 (1985).

${ }^{23}$ C. Haas, Phys. Rev. 168, 531 (1968). 